\title{
Damage of amorphous carbon induced by soft x-ray femtosecond pulses above and below the critical angle
}

\author{
J. Chalupský, ${ }^{1}$ V. Hájková, ${ }^{1}$ V. Altapova ${ }^{2}$ T. Burian, ${ }^{1}$ A. J. Gleeson, ${ }^{3}$ L. Juha, ${ }^{1}$ M. Jurek, ${ }^{4}$ \\ H. Sinn, ${ }^{2}$ M. Störmer, ${ }^{5}$ R. Sobierajski, ${ }^{4}$ K. Tiedtke, ${ }^{6}$ S. Toleikis, ${ }^{6}$ Th. Tschentscher, ${ }^{2}$ \\ L. Vyšín, ${ }^{1}$ H. Wabnitz, ${ }^{6}$ and J. Gaudin ${ }^{2, a)}$ \\ ${ }^{1}$ Institute of Physics, Academy of Sciences of the Czech Republic, Na Slovance 2, 18221 Prague 8, \\ Czech Republic \\ ${ }^{2}$ European XFEL, DESY, Notkestr., 85 D-22607 Hamburg, Germany \\ ${ }^{3}$ CCRLC Daresbury Laboratory, Warrington, Cheshire WA4 4AD, United Kingdom \\ ${ }^{4}$ Institute of Physics, Polish Academy of Sciences, Al. Lotników 32/46, PL-02-668 Warsaw, Poland \\ ${ }^{5}$ GKSS-Forschungszentrum Geesthacht GmbH, Max-Planck-Strasse, 121502 Geesthach, Germany \\ ${ }^{6}$ HASYLAB/DESY, Notkestr., 85 D-22607 Hamburg, Germany
}

(Received 5 June 2009; accepted 1 July 2009; published online 23 July 2009)

\begin{abstract}
We present results of damage studies conducted at the Free Electron LASer in Hamburg (FLASH) facility with $13.5 \mathrm{~nm}(91.8 \mathrm{eV})$ and $7 \mathrm{~nm}(177.1 \mathrm{eV})$ radiations. The laser beam was focused on a sample of 890-nm-thick amorphous carbon coated on a silicon wafer mimicking a x-ray mirror. The fluence threshold for graphitization was determined for different grazing angles above and below the critical angle. The observed angular dependence of $F_{\text {th }}$ is explained by the variation in absorption depth and reflectivity. Moreover, the absorbed local dose needed for the phase transition leading to graphitization is shown to vary with the radiation wavelength. (C) 2009 American Institute of Physics. [DOI: 10.1063/1.3184785]
\end{abstract}

Free-electron lasers (FELs) will deliver femtosecond millijoule light pulses in the $\mathrm{keV}$ photon energy range. The interaction of such light pulses with solids is not yet extensively investigated. This lack of knowledge represents a severe limitation for the design of the x-ray FEL optics. In fact, the requirements of proposed experiments create highly demanding constraints on the x-ray optic elements. It is hence of fundamental importance to understand the interaction mechanisms occurring at the surface of the optical elements.

A first step toward this understanding is to study the interaction of intense radiation using soft x-ray (XUV) FEL sources, as already reported for $32.5 \mathrm{~nm}$ at normal incidence. ${ }^{1}$ The present work extends these investigations in terms of wavelengths down to $7 \mathrm{~nm}$ corresponding to 177.1 $\mathrm{eV}$ photon energy and down to $4.3^{\circ}$ grazing angle. We present results for amorphous carbon $(a-\mathrm{C})$ which is already in use at XUV FEL beamlines ${ }^{2,3}$ and is a possible candidate for future hard X-ray FEL optics. The usual x-ray mirrors work in total external reflection geometry, i.e., the grazing angle is smaller than the critical angle $\theta_{c}$ defined for each wavelength as $\theta_{c}=\sqrt{ }(2 \delta),{ }^{4}$ where $\delta$ is the real part of the refractive index $n=1-\delta+i \beta$. In total external reflection geometry and XUV radiation case (where $\delta$ is positive and $\beta$ is nonzero for materials), the electric field is propagating in the medium along the surface and decays with exponential dependence on depth. ${ }^{4}$ The exponential decay corresponds to an absorption depth, distance normal to the surface, of few nanometers. The damage mechanism in this specific configuration should show differences compared to the normal incidence case. We are reporting of experimental investigations for different angles and wavelengths of the surface damage, defined as any irreversible modification of the optical properties of the initial material, below the ablation threshold.

${ }^{a)}$ Electronic mail: jerome.gaudin@xfel.eu.
The experiment was performed at Free Electron LASer in Hamburg (FLASH), using the setup described in the Ref. 1. The samples were placed in the focus of a $2 \mathrm{~m}$ focal length ellipsoidal mirror of the BL2 beamline. ${ }^{3} \mathrm{~A}$ fast shutter enables to select a single pulse. The pulse energy is measured using a gas ionization detector located before the optical elements of the beamline, for which the total transmission has been measured to be 0.64 (Ref. 3) at $13.5 \mathrm{~nm}$, the same value is assumed at $7 \mathrm{~nm}$. The grazing angle was determined with the following procedure: a charge coupled device (CCD) camera was mounted behind the sample holder in order to measure the direct beam position. Then, the sample was inserted in the beam and the position of the reflected beam was measured on the CCD camera. The distance sample of CCD, noted as $a$, and the measured horizontal shift between the straight and the reflected beam, noted as $b$, give the grazing angle $2 . \alpha=a \tan (b / a)$, with an accuracy of $0.2^{\circ}$. At $13.5 \mathrm{~nm}$, the grazing angles $18.7^{\circ}, 15.1^{\circ}$, and $4.3^{\circ}$ were investigated and $10^{\circ}, 7.7^{\circ}$, and $4.3^{\circ}$ at $7 \mathrm{~nm}$, the critical angle $\theta_{c}$ being $15.8^{\circ}$ for $13.5 \mathrm{~nm}$ and $8^{\circ}$ for $7 \mathrm{~nm}$. For each sample and each angle, at least 60 single shots were recorded.

The samples consist of $890 \mathrm{~nm}$ thick $a$-C coating produced by magnetron sputtering on a $\mathrm{Si}$ wafer. This thickness was chosen to be larger than the attenuation depth at normal incidence in order to minimize the possible influence of the substrate. The mean roughness was measured to be $0.4 \mathrm{~nm}$. The $s p^{3} / s p^{2}$ ratio is a fundamental parameter of the material which has been shown to be directly proportional to the density. ${ }^{5}$ From x-ray reflectivity measurement, ${ }^{6}$ we determined the density to be $2.2 \mathrm{~g} / \mathrm{cm}^{3}$, leading to a $s p^{3}$ fraction of nearly $20 \%$.

The samples were investigated using Nomarski (differential interference contrast) microscope (Fig. 1). Two concentric circles can be distinguished induced by two different damage processes corresponding to material expansion (outer part) and ablation (inner part). The most external 
$20 \mu \mathrm{m}$

FIG. 1. (Color online) Nomarski microscope images of damage by $13.5 \mathrm{~nm}$ beam at normal incidence (left) and $18.5^{\circ}$ grazing angle (right). The scale is the same for both images.

circle corresponds to the lowest damage threshold. Selected spots were also investigated using atomic force microscopy confirming that this damage is a volume expansion. In the following we will focus on this first process which induces degradation of optical properties, therefore being of interest for x-ray FEL optics. The possible process for volume expansion is graphitization of $a$-C, i.e., phase transition from $s p^{3}$ to $s p^{2}$ bonding. This damage phenomenon is general for diamondlike material submitted to intense flux of photons ${ }^{1,7}$ or particles. In the case of $a-\mathrm{C}$, the exact structure of the graphitized material can differ due to the different carbon structures (graphite plane, nanocrystallite, rings ${ }^{8}$ ). Investigations of this aspect are underway but beyond the scope of the present letter.

For each spot, the area of the outer contour was measured and correlated with the measured laser pulse energy. The dependence of the damaged surface to energy is then plotted, as shown in Fig. 2, for the normal incidence case at both wavelengths, in order to determine the damage threshold energy $E_{\mathrm{th}}$. The $13.5 \mathrm{~nm}$ beam has nearly a Gaussian shape and data points were fitted (see Fig. 2) with a logarithmic function, ${ }^{9}$ allowing to determine $E_{\mathrm{th}}$. To retrieve the fluence threshold $F_{\text {th }}$, one needs to determine the beam radius. In the $13.5 \mathrm{~nm}$ case, the radius was measured to be $5 \mu \mathrm{m}$ (1/e radius), using a well established technique. ${ }^{10}$ In the $7 \mathrm{~nm}$ case, the beam shows shape irregularities preventing to use the Liu's method. Data points were then linearly fitted (Fig. 2 ). The beam radius was determined using the $Q$-factor method $^{11}$ which allows retrieving a generalized area in the case of nonGaussian beam. The focused beam spot area was found to correspond to a circle of $4.5 \mu \mathrm{m}$ radius. This procedure is nevertheless less accurate explaining the larger uncertainty on $F_{\text {th }}$.

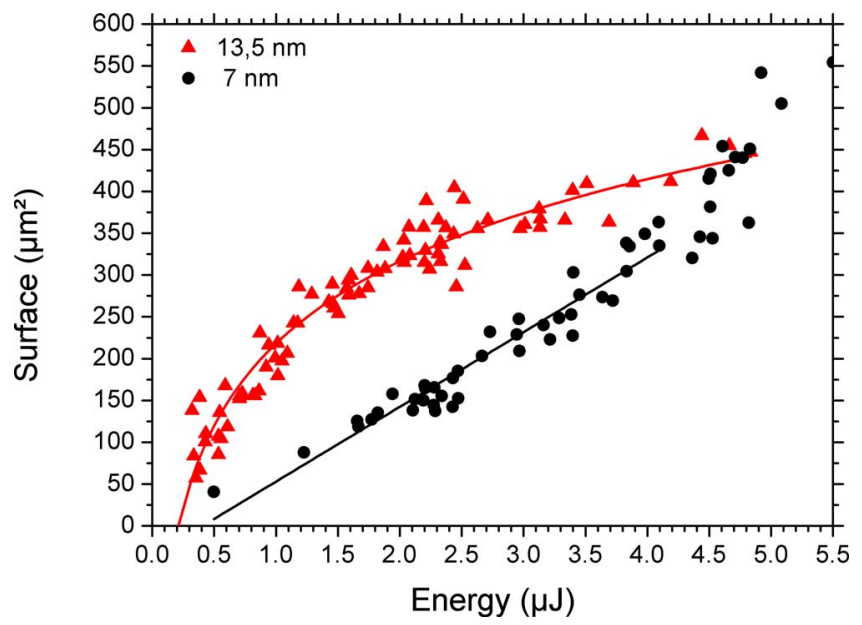

FIG. 2. (Color online) Diameter of damage corresponding to graphitization as a function of the impinging laser pulse energy. Both curves correspond to the normal incidence case. The curves represent fits used to determine $E_{\text {th }}$.

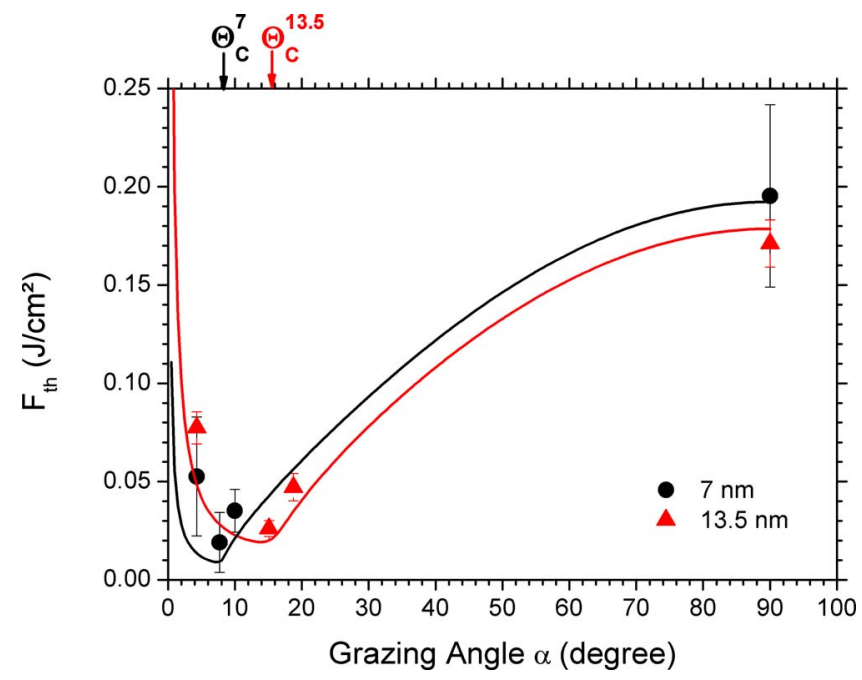

FIG. 3. (Color online) Threshold fluence for damage as a function of grazing angle. The curves show the ratio $d_{a} /(1-R)$, normalized with a constant in order to scale the experimental data. The value of $\Theta_{c}$ is indicated on the top for both wavelengths.

In Fig. 3, the resulting $F_{\text {th }}$ as a function of the grazing angle are given. The values for $13.5 \mathrm{~nm}(7 \mathrm{~nm})$ are $171 \pm 40 \mathrm{~mJ} \mathrm{~cm}^{-2}\left(195 \pm 46 \mathrm{~mJ} \mathrm{~cm}^{-2}\right)$ in the normal incidence case, $47 \pm 7 \mathrm{~mJ} \mathrm{~cm}^{-2}\left(35 \pm 11 \mathrm{~mJ} \mathrm{~cm}^{-2}\right)$ at $18.75^{\circ}$ $\left(10^{\circ}\right), 26 \pm 4 \mathrm{~mJ} \mathrm{~cm}^{-2}\left(19 \pm 23 \mathrm{~mJ} \mathrm{~cm}^{-2}\right)$ for $15.1^{\circ}\left(7.7^{\circ}\right)$, and $77 \pm 8 \mathrm{~mJ} \mathrm{~cm}^{-2}\left(52 \pm 30 \mathrm{~mJ} \mathrm{~cm}^{-2}\right)$ at $4.3^{\circ}$. The curves plotted in Fig. 3 represent the ratio $d_{a} /(1-R), d_{a}$ being the absorption depth and $R$ the reflectivity. ${ }^{12}$ This ratio directly relates the energy density effectively deposited in the material to $F_{\text {th }}$. The trends are very similar to the ones that can be inferred from the measurements and allow explaining the measured data in a very simple manner. From normal incidence to the critical angle, the absorption depth is decreasing (from 160 to $10 \mathrm{~nm}$ for $13.5 \mathrm{~nm}$ and from 632 to $12 \mathrm{~nm}$ for $7 \mathrm{~nm}$ ) while the reflectivity stays very weak below 0.1 . Hence, the energy density increases inducing a decrease of $F_{\text {th }}$. Below $\theta_{c}$, the absorption length stays almost constant as the process of external total reflection occurs. In this case, the value of $d_{a}$ is nearly constant, down to $4 \mathrm{~nm}$ for both wavelengths, while the reflectivity is increasing up to 0.9 for both wavelengths at $4.3^{\circ}$, hence diminishing the energy really absorbed in the material. As a consequence the density of energy decreases and $F_{\text {th }}$ is increasing as shown by the measured point.

In order to get a deeper insight in the physics of the interaction, we calculated the absorbed dose per atom defined as $D_{\mathrm{th}}=E_{\mathrm{th}}(1-R) /\left(S d_{a} n_{a}\right), n_{a}=1.1 \times 10^{23} \mathrm{~cm}^{-3}$ being the atom density and $S$ the focal spot area. Figure 4 presents the calculated dose as a function of $d_{a}$. For both wavelengths, the trend is similar; the dose increases for $13.5 \mathrm{~nm}(7 \mathrm{~nm})$ from $0.60 \pm 0.04(0.13 \pm 0.05)$ to $0.89 \pm 0.13(0.27 \pm 0.08)$, $1.00 \pm 0.16 \quad(0.33 \pm 0.27), \quad$ and finally to $1.09 \pm 0.12$ $(0.69 \pm 0.40) \mathrm{eV} /$ atom. There is a clear increase of $D_{\mathrm{th}}$ as $d_{a}$ is decreasing, meaning that more energy is needed to damage the sample. We interpret this result as an effect of the electrons transport. In fact, in the case of $4.3^{\circ}$ grazing angle, $d_{a}$ is nearly $4 \mathrm{~nm}$ for both wavelengths and the inelastic mean free path in $a$-C (Ref. 13) is in the order of $0.5-1 \mathrm{~nm}$. Before recombining in the valence band, electrons will undergo several collisions and diffuse on a depth larger than $d_{a}$. There- 


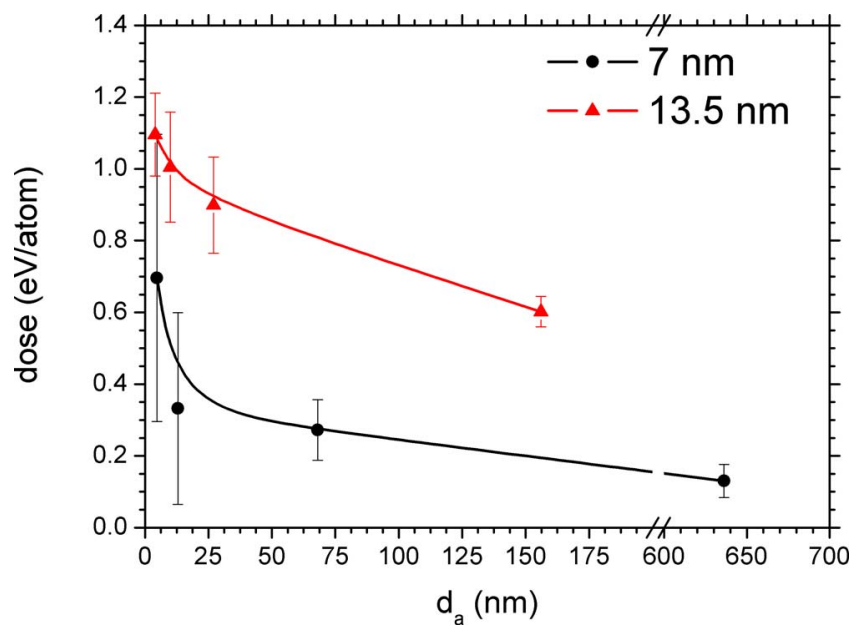

FIG. 4. (Color online) Absorbed dose as a function of absorption depth.

fore, a fraction of the energy is carried away from the effectively irradiated volume by the electrons. This process lowers the amount of energy available for the damage process explaining the observed increase of $D_{\text {th }}$.

Furthermore, Fig. 2 shows that $D_{\text {th }}$ depends on the wavelength; $7 \mathrm{~nm}$ values being lower than for the $13.5 \mathrm{~nm}$ case. Theoretical studies ${ }^{14,15}$ for optical wavelengths have shown that a nonequilibrium distribution of electrons excited by a femtosecond laser pulse results in lowering of the energy barrier between the diamond and graphite structure. This should equally be the case for 7 and $13.5 \mathrm{~nm}$ photons. Here, the $7 \mathrm{~nm}$ radiation should excite a distribution of electrons having an even higher energy tail than for $13.5 \mathrm{~nm}$, while in both cases the same valence band electrons would be ionized. Therefore, the transient energy barrier energy is lowered in the $7 \mathrm{~nm}$ case which also lowers the value of $D_{\text {th }}$ needed for the phase transition. The wavelength dependence can then be explained, in the framework of this model, by the nonthermal origin of the damage observed.

To summarize, we have measured the fluence damage threshold for $a-\mathrm{C}$ at two different wavelengths for grazing angles above and below the critical angle. The present study demonstrates the effects electron transport and the nonthermal origin of the damage process which induces a dependence on the wavelength. If one now considers the hard $\mathrm{x}$-rays range, i.e., photon energy in the $\mathrm{keV}$ range, the effect of electron transport should become more efficient as the inelastic mean free path increase with higher kinetic energy. On the other hand, in the specific case of carbon as the electronic temperature should also increase, the threshold dose for damage should also decrease. Our results also emphasize that considering thermal melting as a threshold for damage, defined as irreversible modification of the optical properties, is at least for carbon not a safe criteria for the design of the beamline.

The authors greatly thank the support of the FLASH scientific and technical team for their help during the beamtime enabling a successful experiment. Financial support from Czech Ministry of Education (Grant Nos. LC510, LC528, and LA08024), Academy of Sciences of the Czech Republic (Grant Nos. KAN300100702, Z10100523, IAAX00100903, and IAA400100701), and Grant Agency of the Czech Republic (Grant No. 202/08/H057) is greatly appreciated.

${ }^{1}$ S. P. Hau-Riege, R. A. London, R. M. Bionta, M. A. McKernan, S. L. Baker, J. Krzywinsky, R. Sobierajski, R. Nietubyc, J. B. Pelka, M. Jurek, L. Juha, J. Chalupský, J. Cihelka, V. Hájková, A. Velyhan, J. Krása, J. Kuba, K. Tiedtke, S. Toleikis, Th. Tschentscher, H. Wabnitz, M. Bergh, C. Caleman, K. Sokolowski-Tinten, N. Stojanovic, and U. Zastrau, Appl. Phys. Lett. 90, 173128 (2007).

${ }^{2}$ B. Steeg, L. Juha, J. Feldhaus, S. Jacobi, R. Sobierajski, C. Michaelsen, A. Andrejczuk, and J. Krzywinski, Appl. Phys. Lett. 84, 657 (2004).

${ }^{3}$ K. Tiedtke, A. Azima, N. von Bargen, L. Bittner, S. Bonfigt, S. Düsterer, B. Faatz, U. Frühling, M. Gensch, Ch. Gerth, N. Guerassimova, U. Hahn, T. Hans, M. Hesse, and K. Honkavaar, U. Jastrow, P. Juranic, S. Kapitzki, B. Keitel, T. Kracht, M. Kuhlmann, W. B. Li, M. Martins, T. Núñez, E. Plönjes, H. Redlin, E. L. Saldin, E. A. Schneidmiller, J. R. Schneider, S. Schreiber, N. Stojanovic, F. Tavella, S. Toleikis, R. Treusch, H. Weigelt, M. Wellhöfer, H. Wabnitz, M. V. Yurkov, and J. Feldhaus, New J. Phys. 11, 023029 (2009).

${ }^{4}$ D. Attwood, Soft X-ray and Extreme Ultraviolet Radiation (Cambridge University Press, Cambridge, 1999).

${ }^{5}$ J. Robertson, Mater. Sci. Eng. R. 37, 129 (2002).

${ }^{6}$ M. Störmer, C. Horstmann, D. Häussler, E. Spiecker, F. Siewert, F. Scholze, F. Hertlein, W. Jäger, and R. Bormann, Proc. SPIE 7077, 707705 (2008).

${ }^{7}$ G. Dumitru, V. Romano, H. P. Pimenov, T. Kononenko, M. Sentis, J. Hermann, and S. Bruneau, Appl. Surf. Sci. 222, 226 (2004).

${ }^{8}$ A. C. Ferrari and J. Robertson, Phys. Rev. B 61, 14095 (2000).

${ }^{9}$ J. M. Liu, Opt. Lett. 7, 196 (1982).

${ }^{10}$ J. Chalupsky, L. Juha, J. Kuba, J. Cihelka, V. Háková, S. Koptyaev, J. Krása, A. Velyhan, M. Bergh, C. Caleman, J. Hajdu, R. M. Bionta, H. Chapman, S. P. Hau-Riege, R. A. London, M. Jurek, J. Krzywinski, R. Nietubyc, J. B. Pelka, R. Sobierajski, J. Meyer-ter-Vehn, A. KrenzTronnier, K. Sokolowski-Tinten, N. Stojanovic, K. Tiedtke, S. Toleikis, T. Tschentscher, H. Wabnitz, and U. Zastrau, Opt. Express 15, 6036 (2007).

${ }^{11}$ J. Chalupsky, J. Krzywinski, L. Juha, V. Hájková, J. Cihelka, T. Burian, L. Vyšín, J. Gaudin, A. Gleeson, M. Jurek, A. R. Khorsand, D. Klinger, H. Wabnitz, R. Sobierajski, M. Störmer, K. Tiedtke, and S. Toleikis, "Spot size characterization of focused non-Gaussian beams" (unpublished).

${ }^{12}$ Centre for X-Ray Optics, http://henke.lbl.gov/optical_constants/.

${ }^{13}$ B. Ziaja, D. van de Spoel, A. Szöke, and J. Hadju, Phys. Rev. B 64, 214104 (2001).

${ }^{14}$ C. Z. Wang, K. M. Ho, M. D. Shirk, and P. A. Molian, Phys. Rev. Lett. 85, 4092 (2000)

${ }^{15}$ H. O. Jeschke, M. E. Garcia, and K. H. Bennemann, Phys. Rev. B 60, R3701 (1999). 